\title{
Collaboration through Digital Integration - An Overview of IT-OT-Integration Use-Cases and Requirements
}

\author{
Jan Hicking, Max-Ferdinand Stroh, Sebastian Kremer \\ FIR, Institute for Industrial Management at RWTH Aachen University, Campus-Boulevard \\ 55, 52074 Aachen, Germany \\ \{Jan.Hicking, Max-Ferdinand.Stroh, Sebastian.Kremer\}@fir.rwth-aachen.de
}

\begin{abstract}
Digitalization and Industry 4.0 continue to shape our industrial environment and collaboration. For many enterprises, a key challenge in moving forward in this matter is the integration of their shop-floor systems (hard- and software) with their office-floor systems to harvest the full potential of industry 4.0. A multitude of different technologies and respective use-cases available on the market leave many companies startled. This paper presents a set of use-cases for IT-OT-Integration to bring transparency into a company's digital transformation. Additionally, a technical requirements profile for integrating ITand OT-Systems based on the use cases is presented. Both, use-cases and their requirements, guide companies in selecting the digitalization measures that fit their current situation and help in identifying technical challenges that need to be addressed in the transformation process.
\end{abstract}

Keywords: Industry 4.0, IT-OT-Integration, Digitalization

\section{Introduction}

Industry 4.0 together with digital transformation pose great innovation opportunities for many different industries and enterprises [1]. A majority of Industry 4.0 use-cases require integrating shopfloor systems, so called OT systems, with an enterprise's office floor systems (IT systems) [2, 3]. Being a central component of Industry 4.0, the integration of IT and OT also becomes a main component for collaborative networks, enabling to harvest their full potential [4].

However, realizing IT-OT-Integration is a main challenge for companies, especially for the limited budgets of SMEs [5-7]. Furthermore most enterprises' IT-OTLandscape, meaning the existing IT-Systems, OT-Systems and interfaces, are very heterogeneous and companies are lacking transparency [8]. In addition, suitable methods for approaching a structured IT-OT-Integration process are missing [9, 10].

To address the issue the authors have developed a systematic approach for IT-OTIntegration based on the as-is assessment of an IT-OT-Landscape as well as a selection of Industry 4.0 utility potentials. Thus, combining a company's digitalization goals with the as is assessment of its infrastructure. 
After a brief introduction into IT-OT-Integration and utility potentials, this paper presents an overview of industry 4.0 (utility) potentials forming the base for a structured IT-OT-Integration process. Next to that, an IT-OT-Integration profile prototype for assessing the status-quo of an IT-OT-Landscape as well as the matching process with the utility potentials is presented.

\section{Developed IT-OT-Integration Methodology}

To tackle the challenges of IT-OT-Integration, both the potential digitalization usecases that are of relevance for a company's digital transformation as well as the existing IT-OT-Landscape need to be considered. This ensures the alignment of a company's strategic goals with its current situation. IT-OT-Integration refers to the interconnection of IT- and OT-Systems $[10,11]$. The term, IT-System refers to the office floor systems of an enterprise such as: ERP (Enterprise Resource Planning) or Customer Relationship Management (CRM) [10, 12]. OT-System refers to the shop floor systems such as machines, scales, scanners and sensors, but also the software included for controlling them, such as MES (Manufacturing Execution System) [10,12]. For structural reasons, MES is considered as an IT-System in terms of the methodology presented in this paper. In their previous work the authors presented a methodology for strategically selecting different digitalization measures [10]. Based on this work, this paper presents the next building blocks within the developed general IT-OT-Integration process. Fig. 1 gives an overview of this process.

In the beginning, potential digitalization measures, the so called utility potentials, are selected. In this paper, the term utility potential is used to describe business benefits (utility) in combination with their digitalization measure (potential) [10]. The selection process is covered in detail in [10]. In section 3 the authors present a list of predefined utility potentials for industrial application, also providing orientation in the process of the digital transformation.

After that, a company's or a certain environment's (such as a disctinct manufacturing line) IT-OT-Landscape is assessed. The methodology uses a morphological box as profile for assessing the as is status of the IT-OT-Landscape. For every system that is part of the assessment a profile is filled and stored for later assessment. Section 4 of this paper gives a detailed description of the assessment profile.

Step three uses the same profile for the matching process. In that process, the profiles from the assessment are compared with the profiles filled with the requirements of the utility potentials, revealing, which of the potentials can be easily fulfilled and which require further integration effort.

Finally, the matching results are combined with a set of action recommendations for starting the integration process. From there on, a company can take individual steps in realizing their IT-OT-Integration potentials. 


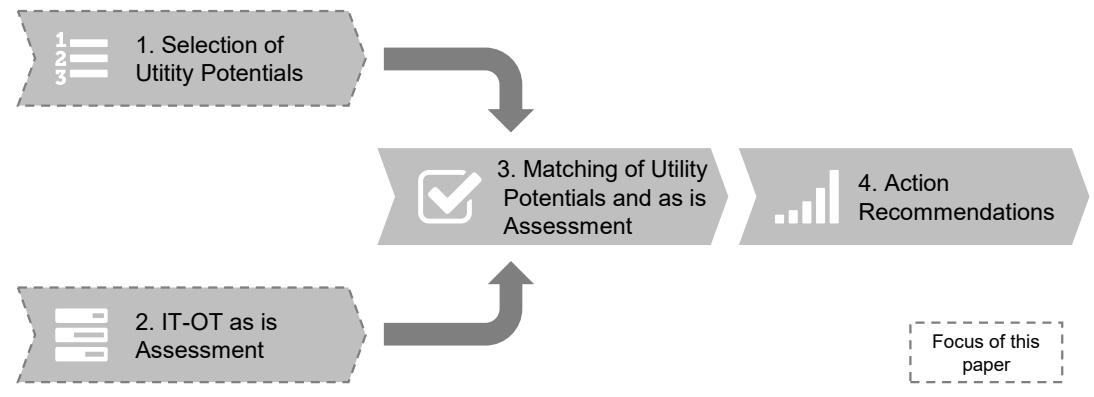

Fig. 1. Overview of developed methodology

\section{Industry 4.0 Utility Potentials}

The presented list of Industry 4.0 utility potentials was developed within a research project which forms the basis for this research. The potentials are derived from both literature research as well as expert interviews and were verified with the members of the user committee of the research project. The main literature sources include [13-18]. The expert interviews yielded individually applicable use cases and their respective analysis in the context of utility potentials.

Utility potentials map to general benefits to be achieved in production, consisting of cost reduction, optimization of process time, enhancement of the product's quality and flexibility of production. They describe specific measures that are classifiable into the categories promotion of transparency, decision support and active production adaption (compare Table 1).

Table 1: List of Utility Potentials clustered into three categories

\begin{tabular}{|c|c|}
\hline \multirow{10}{*}{$\begin{array}{l}\text { Promotion of } \\
\text { Transparency }\end{array}$} & Digital Order Tracking \\
\hline & Digital Worker Guidance (e.g. AR) \\
\hline & Realization of a Digital Twin \\
\hline & Realization of a Digital Shadow \\
\hline & Digital Capture of Lead Time \\
\hline & Realization of Condition Monitoring \\
\hline & Automatic Process Quality Documentation \\
\hline & Automatic Collection of Production KPI \\
\hline & Data-based Derivation of actual Process Cost \\
\hline & Order Status Transparency in Production \\
\hline \multirow{4}{*}{$\begin{array}{l}\text { Decision } \\
\text { Support }\end{array}$} & Predictive Maintenance \\
\hline & Dynamic Pricing in Production \\
\hline & Realization of a Production's Digital Show \\
\hline & Automatic Quality Evaluation with Data Analytics / Machine Learning \\
\hline \multirow{6}{*}{$\begin{array}{c}\text { Active } \\
\text { Production } \\
\text { Adaption }\end{array}$} & Reduction of Machine Downtime \\
\hline & Production Process Optimization with Data Analytics / Machine Learning \\
\hline & Optimization of Process Duration \\
\hline & Active Energy Management using Data Analytics \\
\hline & Adaptive Production Adjustment for Errors and Downtimes \\
\hline & Automatic Machine Configuration based on the Order \\
\hline
\end{tabular}


The category promotion of transparency includes measures, in which data is collected to visualize operational conditions and further allow the analysis of simple causalities between process parameters and the product.

The support of decision-making builds on top of the enhanced transparency, by utilizing further data sources and active analysis to generate insights into production, forecasts and detect necessary actions.

The final category, active production adaption, usually adds onto the previous decision-making, by actively reacting to available information. The adaption takes the form of automatic decision-making and regulation of the process or system. In contrast to the perceived complexity, the measures implementing active adaption can also represent a simple functionality such as the automatic configuration of machines in response to an order command.

The identified utility potentials were each analyzed to determine the required functionalities and architecture within the construct of IT components, OT components and their corresponding connectivity. This analysis was supported by modelling the expected data flow, to demonstrate the individually required system in a network of general IT and OT components, as shown in Fig. 2. The model allowed an intuitive translation of the utility potentials into the proposed IT-OT-Integration Profile.

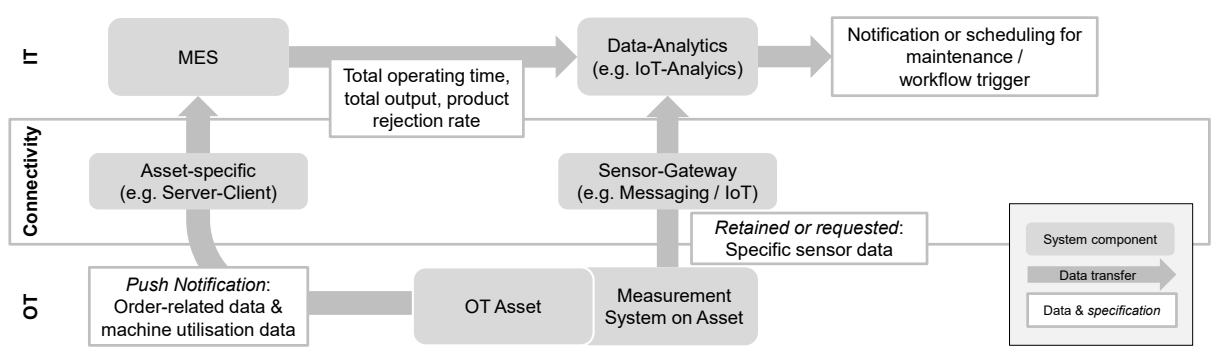

Fig. 2: Requirements on the IT-OT-architecture for the utility potential "Predictive Maintenance"

\section{Prototype of IT-OT-Integration Profile}

The matching of a production's IT-OT as is assessment to the determined utility potentials requires a unified IT-OT-integration profile. The goal is to establish a generalized framework that allows a representation of both actual, specific production systems/architectures and the diffuse requirements of a utility potential, without dictating definitive solutions and technologies. Furthermore, the as is and the proposed architecture need to be comparable to derive technical measures that are required to fulfill the utility potentials requirements.

The proposed profile divides the underlying architecture into OT-components, ITcomponents and interfaces, whereas each specific component in an actual production is represented as its own instance within either of these sections. An ERP-System for example is represented as an IT-component, a production machine as an OT-component and their interconnection as an interface component. The whole structure including the list of options for the components' attributes is shown in Table 2 (table is split into three 
segments due to print formatting). The structure and attributes were derived and developed in expert interviews. The profiles are used to capture the specification of a component, by selecting relevant options of a given attribute.

Table 2: Unified OT-, Interface- and IT-profiles with corresponding attributes and their respective list of options (gray attributes are not defined by utility potentials)

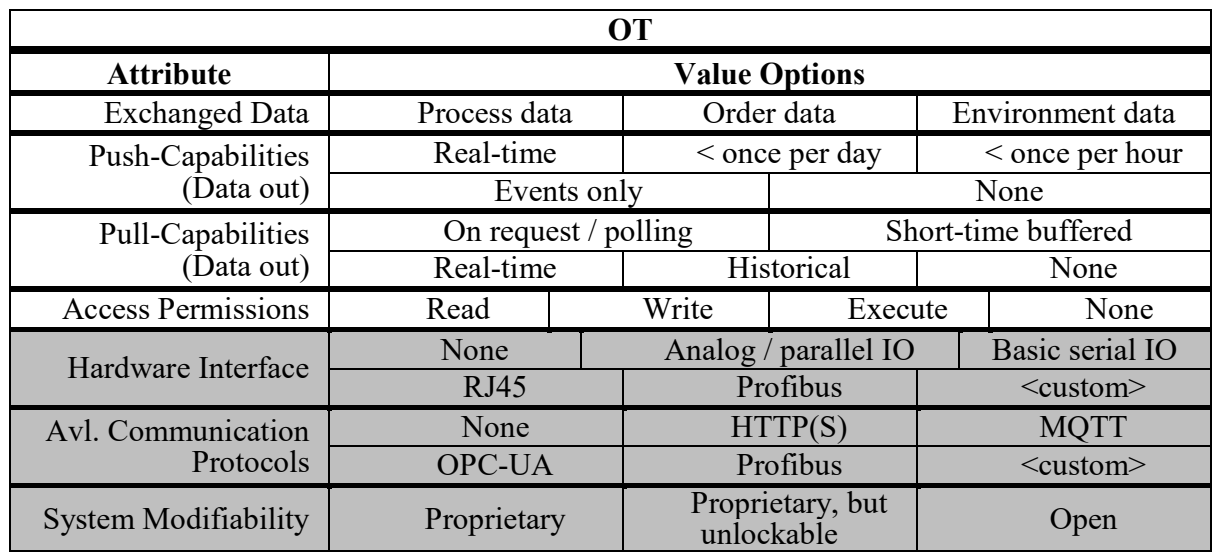

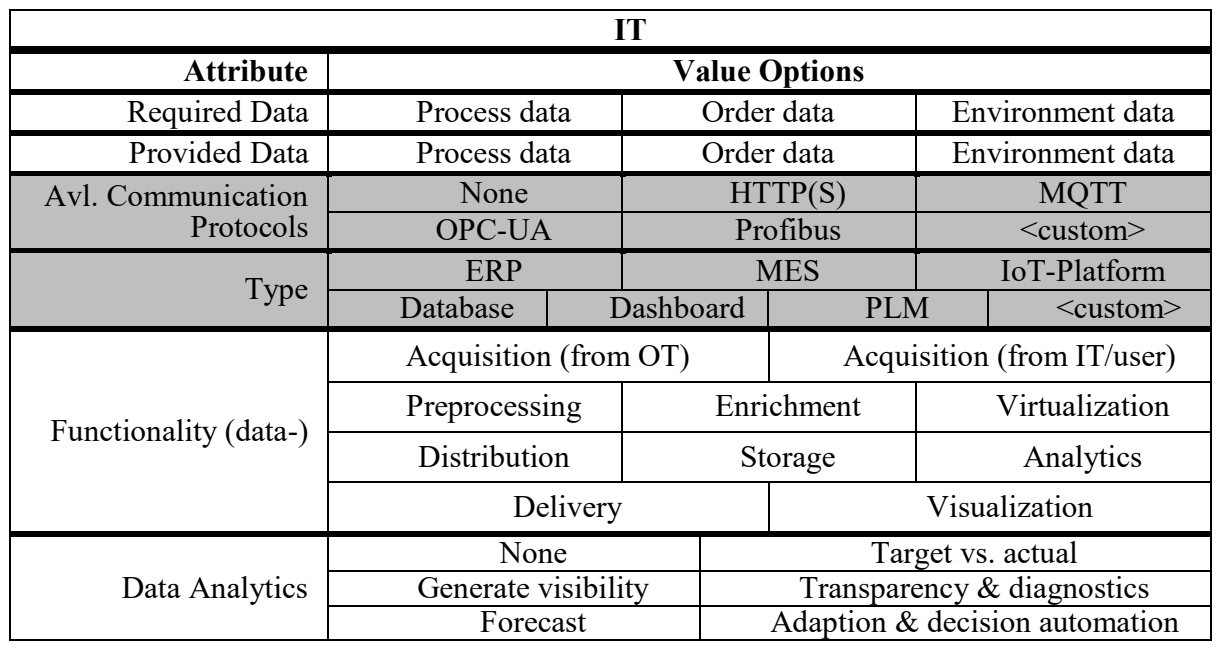




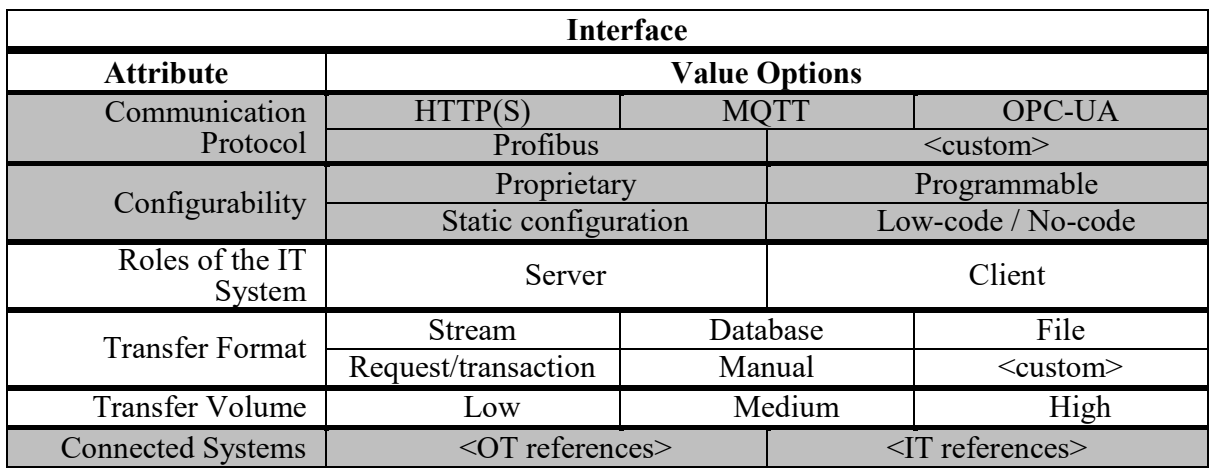

The OT profile comprises the general type of exchanged data (namely order data, process data and environment data), the technical nature of the interfaces, regarding physical ports and data protocols, and the capabilities of the data transfer. It represents the digital capabilities and functionalities of the OT system under consideration, including all its applied additions e.g. sensors and retrofits.

The IT profile comprises the type of IT system at hand, the implemented communication protocols, the exchanged data (similarly to the OT system) and the general functionality extended by eventual data analytics capabilities. The exchanged data is further divided into required and provided data.

Interfaces between each one or more IT and OT systems are defined by its communication protocol, its configurability and the characteristic of the data transfer regarding directionality, format and bandwidth. Additionally, the interface references the IT and OT systems it is connecting. Multiple interfaces of similar nature, e.g. a oneto-many connection, can easily be summarized within one interface instance by referencing multiple IT or OT components.

Table 3: Attribute-wise matching of as-is assessment (crosses) and chosen utility potential (circles) in an exemplary OT profile

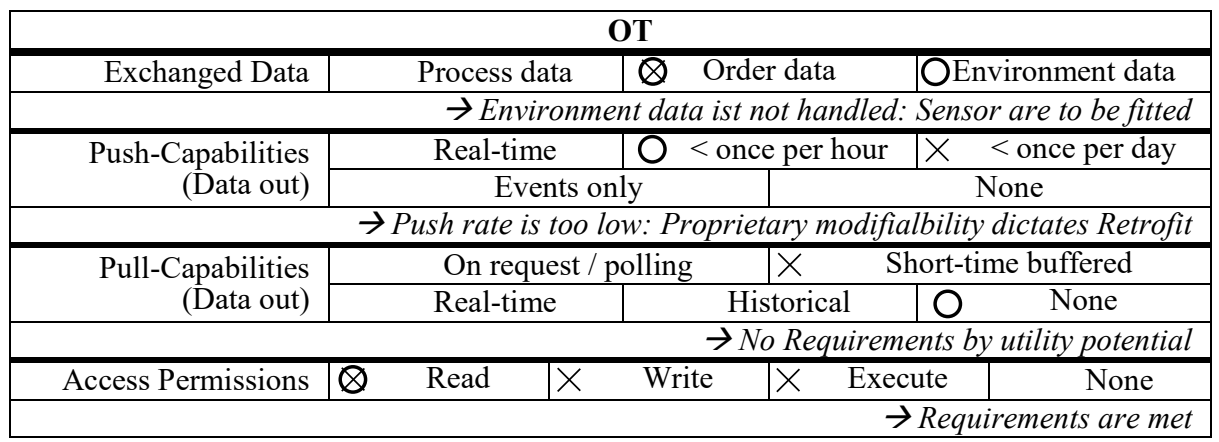

The generalized requirements of a utility potential are summarized in exactly three components, one of each type. The specific attributes of each component are disregarded (marked in gray), as they dictate a technical solution. This approach was chosen to allow for a general evaluation of multiple and vastly different specific production architectures. In fact, disregarded attributes only serve to evaluate possible interconnections of components and eventually the difficulty of achieving such. The 
matching of a production's as-is assessment to the utility potentials is demonstrated in Table 3.

By assessing an attribute's delta to the desired utility potential and evaluating its difficulty of solving the delta, using the specific attributes (in grey), an appropriate measure can be derived. Similarly the interfaces, the IT-systems and the interconnectivity is handled. The overall assessment then allows for an identification of critical measures and their respective difficulties, e.g., solvable by using Retrofits, to achieve the utility potential.

\section{Outlook \& Conclusion}

Mastering the IT-OT-Integration process is a challenge for many companies, especially SMEs. Therefore, this paper presents a set of utility potentials as well as an IT-OTIntegration profile to structure and assist the integration process.

In the beginning, the developed IT-OT-Integration approach is presented and put into the context of previous research activities. Afterwards a set of utility potentials to select in the beginning of an IT-OT-Integration project is shown. Subsequently, an Integration profile with the categories IT, OT and Interface to assist the structured as is assessment of an IT-OT-Landscape is introduced. Finally, the matching process between the selected utility potentials and the as is assessment of the IT-OT-Landscape is explained.

Future research will further explore the presented approach to prove its validity. Additionally, the methodology will be assisted by a web-based application to facilitate the access to IT-OT self-assessments.

\section{Acknowledgements}

The IGF project 20768 BG of the Research Association FIR e. V. at the RWTH Aachen University is funded via the AiF within the framework of the programme for the funding of cooperative industrial research (IGF) by the Federal Ministry of Economics and Energy (BMWi) on the basis of a resolution of the German Bundestag.

\section{References}

1. Vogel-Heuser, B., Bauernhansl, T., Hompel, M. ten (eds.): Handbuch Industrie 4.0 Bd.2. Automatisierung. Springer Vieweg (2017)

2. Bildstein, A., Seidelmann, J.: Migration zur Industrie- 4.0-Fertigung. In: Vogel-Heuser, B., Bauernhansl, T., Hompel, M. ten (eds.) Handbuch Industrie 4.0 Bd. 1. Produktion, pp. 227242. Springer Vieweg, Berlin (2017)

3. Schlick, C. (ed.): Megatrend Digitalisierung - Potenziale der Arbeits- und Betriebsorganisation. GITO, Berlin (2016)

4. Camarinha-Matos, L.M., Fornasiero, R., Afsarmanesh, H.: Collaborative Networks as a Core Enabler of Industry 4.0. In: Collaboration in a Data-Rich World, 506, pp. 3-17. 
Springer International Publishing, Cham (2017) https://doi.org/10.1007/978-3-319-6515141

5. Henke, N., Bughin, J., Chui, M., Manyika, J., Saleh, T., Wiseman, B. and Sethupathy, G.: The age of analytics. Competing in a data driven world

6. Saam, M., Viete, S., Schiel, S.: Digitalisierung im Mittelstand. Status Quo, aktuelle Entwicklungen und Herausforderungen. Frankfurt (2016)

7. Pettey, C.: When IT and Operational Technology Converge. CIOs and IT leaders will drive significant competitive advantages with this integration.

8. Urbach, N., Ahlemann, F.: Die IT-Organisation im Wandel. Implikationen der Digitalisierung für das IT-Management. HMD Praxis der Wirtschaftsinformatik 54, 300312 (2017)

9. Lara, P., Sánchez, M., Villalobos, J.: Bridging the IT and OT Worlds Using an Extensible Modeling Language. In: Comyn-Wattiau, I. (ed.) Conceptual modeling. 35th International Conference, ER 2016, Gifu, Japan, November 14-17, 2016 : proceedings, 9974, pp. 122 129. Springer, [Cham] (2016)

10. Günther Schuh, Hicking, J., Jordan, F., Stroh, M.-F., Saß, S.-A.: Strategic target system to select digitalization measures in manufacturing companies. In: Camarinha-Matos, L.M., Afsarmanesh, H., Ortiz, A. (eds.) Boosting Collaborative Networks 4.0, pp. 227-237. Springer International Publishing, Cham (2020)

11. Phani Kumar Garimella: IT-OT Integration Challenges in Utilities. October 25th -27 th, 2018, Kathmandu, Nepal : an IEEE Nepal Sub Section Conference. IEEE, Piscataway, NJ (2018)

12. Noronha, A., Moriarty, R., O'Connell, K. and Villa, N.: Attaining IoT Value: How To Move from Connecting Things to Capturing Insights. Gain an Edge by Taking Analytics to the Edge, https://www.cisco.com/c/dam/en_us/solutions/trends/iot/docs/iot-data-analyticswhite-paper.PDF

13. Goto, S., O. Yoshie, and S. Fujimura.: Industrial IoT Business Workshop on Smart Connected Application Development for Operational Technology (OT) System Integrator. IEEE IEEM2017 : 10-13 Dec, Singapore. IEEE, Piscataway, NJ (2017)

14. Global Lighthouse Network:. Insights from the Forefront of the Fourth Industrial Revolution (2019)

15. Reinhart, G. (ed.): Handbuch Industrie 4.0. Geschäftsmodelle, Prozesse, Technik. Hanser, München (2017)

16. Kleinemeier, M.: Von der Automatisierungspyramide zu UnternehmenssteuerungsNetzwerken. In: Vogel-Heuser, B., Bauernhansl, T., Hompel, M. ten (eds.) Handbuch Industrie 4.0 Bd. 1. Produktion, pp. 219-226. Springer Vieweg, Berlin (2017)

17. Lechler, A., Schlechtendahl, J.: Steuerung aus der Cloud. In: Vogel-Heuser, B., Bauernhansl, T., Hompel, M. ten (eds.) Handbuch Industrie 4.0 Bd. 1. Produktion, pp. 6174. Springer Vieweg, Berlin (2017)

18. Pelino, M., Hewitt, A.: The Forrester Wave ${ }^{\mathrm{TM}}$ : IoT Software Platforms, Q4 2016. The 11 Providers That Matter Most And How They Stack Up (2016) 